\section{Current research on respiratory viral infections: XIII International Symposium on Respiratory Viral Infections: part 1}

Matthijs RA Welkers ${ }^{\dagger 1}$, Tom Sutherland ${ }^{2}$, Albert DME Osterhaus ${ }^{3}$, Arnold Monto ${ }^{4}$, Menno D de Jong' \& Frederick G Hayden ${ }^{2,5}$

'Department of Medical Microbiology, Academic Medical Centre, Amsterdam, The Netherlands

International Activities, Wellcome Trust, London, UK

3Department of Virology, Erasmus Medical Centre, Rotterdam, The Netherlands

4Department of Epidemiology, University of Michigan School of Public Health, Ann Arbor, MI, USA

EUniversity of Virginia, Division of Infectious Diseases \& International Health, Charlottesville, VA, USA

†Author for correspondence: mrawelkers@gmail.com

\section{International Symposium on Respiratory Viral Infections}

Rome, Italy, 13-16 March 2011

The XIII International Symposium on Respiratory Viral Infections was convened by The Macrae Group LLC (NY, USA) in Rome, Italy, on the 13-16 March 2011. This annual symposium provides a forum for public health specialists, vaccinologists, clinicians, virologists and pharmacologists to discuss recent advances in respiratory virus research in an interdisciplinary fashion. Here, we highlight presentations at this conference with a special focus on influenza.

\section{Surveillance of the}

\section{animal-human interface}

A clear lesson learned from the 2009 H1N1 pandemic and emphasized by many presenters was the importance of surveillance in both humans and animals for the detection of novel pathogens with pandemic potential. Of note, the ancestors of the pandemic H1N1 virus (pH1N1) had been shown to circulate in swine for at least a decade, but phylogenetic analysis indicated that the individual gene segments had not been sampled for intervals of 9-17 years in surveillance studies [1]. Despite the need for data on circulating swine influenza viruses (SIVs), its acquisition is often difficult, largely because of perceived economic threats to the swine industry. In order to address this problem, Malik Peiris of the University of Hong Kong and colleagues have conducted surveillance in Hong Kong's main abattoir since 1998 [2]. Of the 4000-5000 pigs slaughtered there daily, the vast majority are imported from different parts of southern China, so that surveillance can detect viruses from a wide geographic area. Full genome sequencing of isolated SIVs has shown increased diversity related to introductions, reassortment events and cocirculation of multiple SIV lineages since 2002. In addition to $\mathrm{pH} 1 \mathrm{~N} 1$ transmission between pigs, sampling identified different $\mathrm{pH} 1 \mathrm{~N} 1$ viruses, suggesting repeated independent introductions from humans into swine. These findings highlight both the potential for emergence of novel SIVs and the value of systematic abattoir surveillance for detecting SIVs.

Several models including replication patterns in ex vivo human respiratory organ cultures (tissues from upper airways, bronchi and lungs) and transmission in animal models have been used to assess potential SIV transmission risks to humans. When the transmissibility of various viruses was measured in the ferret model, the seasonal $\mathrm{H} 1 \mathrm{~N} 1$ and $\mathrm{pH} 1 \mathrm{~N} 1$ viruses were efficiently transmitted through the aerosol route, but the classical or North American SIVs were transmitted only by direct contact. Interestingly, one previously isolated pandemiclike reassortant, only differing in the neuraminidase $(N A)$ gene, transmitted inefficiently via the aerosol route, but introduction of the $\mathrm{pH} 1 \mathrm{~N} 1$ NA led to highly efficient aerosol transmission. Peiris concluded that global collaboration on enhancing systematic SIV sampling combined with human surveillance at the swine-human interface, whole genomic sequencing and rapid data sharing are needed.

The interplay of economic forces and influenza spread within the swine population in the USA was discussed by Martha Nelson from the Fogarty International Centre (MD, USA). As a consequence of changes in rearing practices within the US swine industry, the directional spread of SIVs has changed nationally. Previously, corn grown in the midwest was transported to

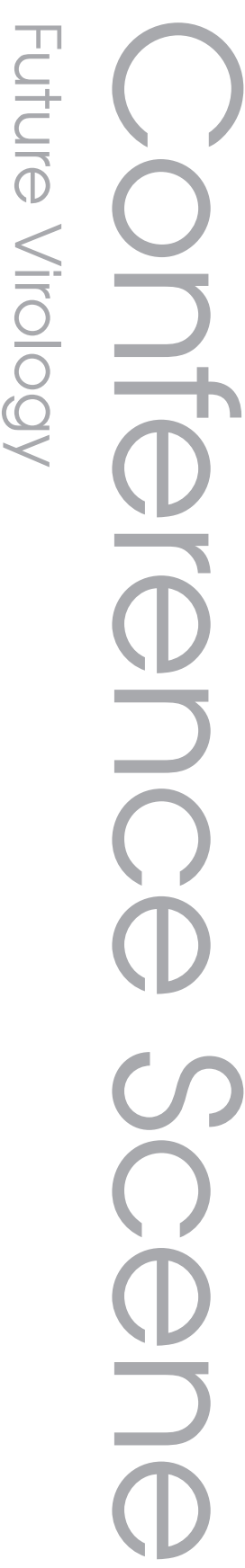

\section{Keywords}

- epidemiology = influenza surveillance $=\mathrm{pH} 1 \mathrm{NI}$

- treatment $\|$ vaccines

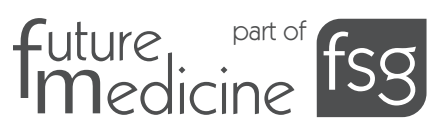


the major pig-farming areas in the southeast and south-central USA. In the 1990 s economic considerations led portions of the swine industry to transport swine from the southeast and southcentral areas into the midwestern 'corn-belt'. This change in approach was shown to be responsible for the observed SIV sequence diversity in a dataset containing 1516 HA1 sequences from the 2003-2010 timeframe, including 41 sequences of $\mathrm{pH} 1 \mathrm{~N} 1$ viruses that were transmitted back into the swine population [3].

The surveillance of avian influenza viruses (AIVs) continues to be a priority, as avian $\mathrm{H} 5 \mathrm{~N} 1$ viruses continue to cause outbreaks in poultry and sporadic severe human infections, and other AIVs remain threats. Ilaria Capua, from the Instituto Zooprofilattico Sperimentale delle Venezie, Italy, showed that four distinct groups (A, B, C and D) of avian H9N2 viruses were cocirculating within 11 central Asian and middle eastern countries, indicating multiple introductions of H9N2 in this geographical region [4]. Extensive intra-subtype reassortant $\mathrm{H} 9 \mathrm{~N} 2$ viruses have been identified, as have inter-subtype reassortants. For instance, the NS gene segment of H9N2 viruses from Pakistan showed evidence of reassortment with HPAI H5N1 viruses belonging to clade 2.2, and with HPAI H7N3 viruses from Pakistan.

Another example of genetic change in $\mathrm{H} 5 \mathrm{~N} 1$ viruses was the finding of two distinct genetic groups ( $\mathrm{A}$ and $\mathrm{B}$ ) that differed in population dynamics and evolutionary rate among clade 2.2.1 H5N1 viruses in Egypt. The clade B viruses circulating in poultry where vaccination has been used extensively had a much higher evolutionary rate compared with clade $A$. Interestingly, the genetic evolutionary rate of all $\mathrm{H} 5 \mathrm{~N} 1$ viruses in Egypt showed a sharp increase 1.5 years after the start of poultry vaccination programs, and sequence analysis showed that changes occurred primarily at predicted antigenic sites [5]. A comparison of $\mathrm{H} 5 \mathrm{~N} 1$ evolutionary rates found significantly higher rates in vaccinating than in nonvaccinating countries. While the impact of vaccination cannot be directly determined, the data are striking in showing much more rapidly evolving influenza virus populations in countries with $\mathrm{H} 5$ vaccine programs. Capua concluded that the prediction models to determine pandemic potential need to be improved, data has to be shared, and simultaneously, the surveillance capacities in animals should be increased to enhance early warning systems.

Kirill Sharshov of the State Research Centre of Virology and Biotechnology (Novisibirsk, Russia) highlighted the unique ecology of western Siberia with its many bird species, migration routes, lakes and rivers, which could serve as a major mixing location for AIVs. During surveillance conducted in 2010, 32 viruses ( $4.3 \%$ of samples) were isolated from four different duck species. Within Siberia, there have been several AIV outbreaks in which water bodies seemed to play a major role. In particular, $\mathrm{H} 5 \mathrm{~N} 1$ outbreaks with different subclades originating from the Uvs-Nuur Lake have occurred; during 2005-2007 only clade 2.2 viruses were isolated, but from 20082010 only clade 2.3 .2 viruses were identified [6]. The reason for this subclade change is unclear, but hemagglutination inhibition (HI) antibody data indicated that the affected avian host species had no preexisting immunity to the new 2.3.2 clade but substantial immunity to 2.2 clade viruses. Therefore, Sharshov suggested that the subclade change might have occurred due to the pressure of population immunity in the natural host species.

\section{Epidemiology \& response to influenza outbreaks}

The keynote lecture was given by Arnold Monto (University of Michigan, IL, USA), who questioned to what extent the distinctions drawn between pandemic and seasonal or nonpandemic influenza make sense. Even though there may be different public health and societal responses to pandemic and inter-pandemic influenza, the divide that has been created, especially in political and funding contexts, is in many ways artificial. Key conceptual problems at present include determining a broadly agreed definition of a pandemic and whether measures of severity, in addition to transmissibility, should be incorporated. When the 2009 pandemic struck, the WHO was still in the process of developing a severity index. The US government had adopted a five-point severity scale, similar to that used for hurricanes, primarily because in the wake of the hurricane Katrina disaster, officials thought that the system would be familiar to the public.

Pandemic preparedness has occurred largely in response to the threat of $\mathrm{H} 5 \mathrm{~N} 1$, which still influences decision-making. Monto stated that examination of historical outbreaks, while useful, could also be misleading. For instance, fear of a 1918-type sequence of events fuelled some media speculation in 2009 that the relatively mild first wave of $\mathrm{pH} 1 \mathrm{~N} 1$ would be followed by massively increased mortality in a second wave. While some reports point out that pandemics since 1918 have been increasingly mild, there are currently insufficient time points to draw conclusions, as each event is unique and 
improved medical treatment and preparedness are important variables. In summary, Monto stated that the current $\mathrm{pH} 1 \mathrm{~N} 1$ virus emerged suddenly, was novel and of animal origin, and transmitted both extensively and rapidly on a global basis, thus fulfilling the current definition of a pandemic virus. Going forward, he highlighted the need for severity categories to be established, so that early in a pandemic, the possible impact and associated responses could be communicated effectively with the public.

An important issue in assessing outbreak impact is how to estimate the severity in regions and populations where surveillance is not well developed. Marc-Alain Widdowson of the International Epidemiology and Research Team (US CDC) presented the preliminary estimates of pandemic 2009 influenza mortality globally and in lower-resource settings. The objective of the study was the timely estimate of the influenzaassociated mortality during the first full year after virus introduction in each country. In order to correct for differences in risk of death due to influenza across countries or regions, the mortality multiplier was introduced. A mortality multiplier is estimated from CDC global surveillance studies and uses the relative rate ratio (compared with the USA) of the risk of dying from a respiratory infection to correct the observed mortality of $\mathrm{pH} 1 \mathrm{~N} 1 \mathrm{in}$ an individual country across different age groups. Using this technique, the estimated pH1N1 mortality after one year of circulation accounted for a median total of 267,000 deaths, with countries in Africa and Southeast Asia accounting for $59 \%$ of these deaths. In contrast, laboratory-confirmed deaths reported to WHO indicated 18,500 deaths globally with the lowest percentage in these regions. The estimates of pH1N1 mortality burden were similar to global seasonal influenza, but Widdowson noted that this comparison is misleading because different methods were used and, contrary to typical seasonal influenza, most pH1N1 mortality occurred in the nonelderly adults, so that life-years lost were considerable. In terms of life-years lost some pH1N1 estimates were similar to the 1968 Hong Kong influenza pandemic [7].

\section{Clinical \& host factors}

Although most $\mathrm{pH} 1 \mathrm{~N} 1$ infections were selflimited, uncomplicated events, the risk of severe illness and mortality was observed to be approximately 7 and 8 times higher, respectively, in children, and 4 and 12 times greater, respectively, in adults aged 18-64 years compared to seasonal influenza [8]. Tim Uyeki from the Influenza
Division of the CDC (GA, USA) described a retrospective observational cohort study from the Pediatric Acute Lung Injury and Sepsis Investigator's (PALISI) network that included 838 critically ill children with confirmed or probable $\mathrm{pH} 1 \mathrm{~N} 1$ infection. In this analysis of patients admitted to one of the PALISI intensive care units (ICUs) from April 2009 until April 2010, the median age was 6 years and more than $70 \%$ had more than one underlying medical condition. The mean time from disease onset to pediatric ICU admission was 4 days, and $64 \%$ of patients presented with lower respiratory tract disease and hypoxia, with 58\% receiving ventilation at admission. Viral coinfections were found in $4.8 \%$ of cases and bacterial coinfection in $38 \%$. The fatal cases had higher Pediatric Risk of Mortality III scores at admission compared with survivors. Other significant mortality risk factors at pediatric ICU admission were leukopenia, neutropenia, thrombocytopenia, elevated creatinine and severe hypoxia. In the multivariate analysis, a significant mortality risk was observed for patients with preexisting neurologic conditions, immunocompromise, presumed MRSA pneumonia, female gender and presence of encephalitis or myocarditis. In previously healthy children the only risk factor was Staphylococcus aureus pneumonia within $72 \mathrm{~h}$ of admission. Uyeki concluded that, despite limitations, this retrospective study provides important information on risk factors and clinical course in critically ill children with pH1N1.

But how does this compare in relation to other severe influenza infections like $\mathrm{H} 5 \mathrm{~N} 1$ ? Menno de Jong from the Academic Medical Centre in Amsterdam addressed this issue in his comparison of severe $\mathrm{H} 5 \mathrm{~N} 1$ and $\mathrm{pH} 1 \mathrm{~N} 1$ infections. Even though the 2009 H1N1 pandemic took much attention away from $\mathrm{H} 5 \mathrm{~N} 1$, the latter continues to cause poultry outbreaks and sporadic human cases, and is thought to be endemic in multiple countries including Vietnam, Cambodia, Egypt and Indonesia. In 2010 alone, poultry outbreaks were described in 16 countries and 48 human cases were reported. Of the $528 \mathrm{H} 5 \mathrm{~N} 1$ patients reported to date, 311 died (59\%), with mortality ranging from $32 \%$ in Egypt to $83 \%$ in Indonesia [101]. In H5N1 infections there is strong evidence for a genetic link, as seen by the almost exclusive involvement of blood relatives in clusters and the very low seroprevalence in most studies of exposed persons. Thus, subclinical H5N1 infections have been infrequently documented and severe disease predominates in $\mathrm{H} 5 \mathrm{~N} 1$ infections, while for 
pH1N1 severe disease occurred in only a small fraction of all infections. Severe pH1N1 illness has developed mostly in patients with underlying chronic comorbidities, pregnancy, and/or obesity, whereas most patients with $\mathrm{H} 5 \mathrm{~N} 1$ illness have had no underlying conditions. Clinically, H5N1 illness presents primarily with viral pneumonia, usually with no secondary bacterial infection and is often associated with sepsis syndrome and/or multi-organ failure. Bacterial coinfections and exacerbations of underlying airway disease have been more common with pH1N1. The principal cause of death has been respiratory failure in both $\mathrm{pH} 1 \mathrm{~N} 1$ and $\mathrm{H} 5 \mathrm{~N} 1$ illness due to progressive viral pneumonia and lung injury with similar histopathology in both types of disease. The correlates of severe disease are similar for both viruses, as the respiratory viral load is higher, viral RNA is more often detectable in blood, and higher plasma cytokine levels correlating with respiratory virus load are observed in severe/fatal disease. $\mathrm{H} 5 \mathrm{~N} 1$ is a highly virulent virus, with the intrinsic ability to cause severe disease in multiple species, while $\mathrm{pH} 1 \mathrm{~N} 1$ virus lacks the virulence characteristics of $\mathrm{H} 5 \mathrm{~N} 1$. However, there are likely to be host genetic predispositions for both viruses with respect to infection by $\mathrm{H} 5 \mathrm{~N} 1$ and to uncommon cases of severe viral pneumonia in $\mathrm{pH} 1 \mathrm{~N} 1$ infections. Professor de Jong concluded with the reminder that $\mathrm{H} 5 \mathrm{~N} 1$ presents a continuing threat to animal and human health.

The immunopathogenesis of severe human influenza remains incompletely understood. $\mathrm{H} 5 \mathrm{~N} 1$ has been previously shown to cause a dysregulation of innate immune responses with excessive proinflammatory cytokine production, but this aspect of pathogenesis is unclear for infections by other influenza variants [9]. Nelson Lee from the Chinese University of Hong Kong (China) described a prospective, 2-year observational study of cytokine and chemokine responses in those hospitalized with either pH1N1 or seasonal influenza. The 63 patients with $\mathrm{pH} 1 \mathrm{~N} 1$ infection were generally younger (mean: 42.8 vs 70.5 years), less frequently had underlying conditions (30 vs $53 \%$ ), and more frequently had severe pneumonia $(54.0$ vs $28.3 \%$ ) leading to a higher ICU admission rate (25.4 vs $1.9 \%$ ) and death (3.2 vs 0\%) compared with the 53 patients with seasonal influenza. Large increases in plasma IL-6, sTNFR-1 and IL-10 levels, but suppression of IP-10, IL-17 were observed in $\mathrm{pH} 1 \mathrm{~N} 1$ cases. Serial samples indicated that the proinflammatory cytokines were persistently activated in $\mathrm{pH} 1 \mathrm{~N} 1$ patients throughout the course of infection, whereas in seasonal infection patients, plasma levels fell more quickly along with the viral load. A high IL-6 concentration was associated with ICU admission and other clinical markers like fever, tachypnea, oxygen desaturation and length of hospital stay. In seasonal influenza patients, plasma IL-6 was also higher in patients with respiratory complications. These findings suggest that upregulated proinflammatory cytokine responses are a characteristic of severe influenza infection and that significant associations exist between cytokine responses, disease severity and clinical outcome. In addition, severe $\mathrm{pH} 1 \mathrm{~N} 1$ may be associated with inadequate Th1-type responses. Lee postulated that the hyperactivation of cytokines is driven by persistently active viral replication, so that antivirals should remain an essential treatment modality, but there might be a role for anti-inflammatory agents.

The role of host factors and more specifically the interplay between pathogen and host in determining the clinical outcome of influenza has not yet been elucidated. Jake Dunning of the Imperial College London, UK, presented preliminary results from the Mechanisms of Severe Acute Influenza Consortium study of pathogenesis in hospitalized adults and children. The study enrolled 257 patients from 11 hospitals in London and Liverpool between December 2009 and February 2011. Of these, 166 had a reverse transcriptase-PCR-confirmed influenza infection, of which $80 \%$ was $\mathrm{pH} 1 \mathrm{~N} 1$. Of the 128 adults with influenza (not exclusively pH1N1), 20\% had no comorbidities, $24 \%$ were asthmatic and $23 \%$ were immunosuppressed (including 13\% HIV-positive). A total of 40 adult patients (31\%) were admitted to ICU, $25 \%$ were intubated or received extracorporeal membrane oxygenation and $9 \%$ died. Of the 34 children 55\% required ICU admission; 29\% of children having chronic neurologic disease as comorbidity. Bacterial coinfections were identified in $16 \%$ of cases, more often $(22 \%)$ in ICUadmitted or fatal cases and less often $(13 \%)$ in survivors. Studies in progress will characterize the viruses detected, host innate and adaptive immune responses, genomics and bacterial flora by deep sequencing of $16 \mathrm{~S}$ RNA.

\section{Influenza treatment}

The standard treatment in serious influenza infection in most countries is currently oral oseltamivir but the optimal dose regimen is uncertain. Rogier van Doorn of the Oxford University Clinical Research Unit in Ho Chi Minh City, 
Vietnam presented the results of a doubleblinded, randomized controlled study performed through the Southeast Asia Infectious Diseases Clinical Research Network in which the standard dose of oseltamivir was compared with a double dose in hospitalized children and adults. Among 311 enrolled patients ( $78 \%$ children), $43 \%$ were infected with $\mathrm{H} 3 \mathrm{~N} 2,20 \%$ with $\mathrm{pH} 1 \mathrm{~N} 1$ and $17 \%$ with influenza $B$ viruses. The median length of illness before the start of therapy was 5 days, and $14 \%$ of patients were admitted to ICU at admission. No differences in virologic or clinical measures, including 28 -day mortality ( $3 \%$ overall), were observed between the groups receiving the standard or double dose of oseltamivir. Adverse and severe adverse events were also similar in both treatment groups. In short, the largest controlled trial in severe influenza to date showed no important clinical or virological benefit from a double dose of oseltamivir.

Progress in clinical testing of new anti-influenza drugs has been substantial. Norio Sugaya from Keiyu Hospital (Yokohama, Japan) presented an update on inhaled laninamivir. Laninamivir is an inhaled NA inhibitor, very similar to zanamivir in its antiviral spectrum but active for longer periods in the respiratory tract. One doubleblind, randomized, oseltamivir-controlled trial during the 2008-2009 influenza season included 184 seasonal H1N1-infected children, of whom almost all had oseltamivir-resistant viruses that were still sensitive to laninamivir [10]. Single doses of laninamivir 20 or $40 \mathrm{mg}$ significantly reduced time to alleviation of illness in this subset by 66.2 and $60.9 \mathrm{~h}$, respectively, and shortened the duration of fever compared with oseltamivir [10]. For unexplained reasons, in a parallel study including 996 adults, no clinical differences were observed between the oseltamivir- and laninamivir-treated patients who had infections with oseltamivirresistant seasonal H1N1 viruses [11]. These studies show that a single-dose inhaled laninamivir treatment was at least comparable to a five day oseltamivir regimen in outpatients. This agent has been approved for treatment of uncomplicated influenza in Japan. Further studies of its effectiveness are in progress.

Peramivir is an intravenously administered neuraminidase inhibitor, already approved for single-dose outpatient treatment in Japan and South Korea. Mike Ison from Northwestern University, IL, USA, described a study comparing intravenous peramivir $300 \mathrm{mg}$ twice a day to intravenous peramivir $600 \mathrm{mg}$ once a day in patients hospitalized with suspected or confirmed influenza infections. Among 234 patients enrolled, only 57 patients (300 $\mathrm{mg}$ dose) and 70 patients (600 mg dose) had positive cultures or reverse transcriptase-PCR, of whom approximately three-quarters had $\mathrm{pH} 1 \mathrm{~N} 1$. No differences were observed in decreases in upper respiratory viral titers or RNA levels over time or clinical outcomes between the groups. Of note, titers of viral RNA were generally $1-2 \log _{10}$ higher and detectable for 24-48 h longer than those of infectious virus. Adverse events were noted in $76 \%$ of the population, most commonly gastrointestinal events (37\%), but these did not differ by treatment. Patients requiring oxygenation at baseline had longer time to clinical resolution compared with patients who did not require oxygenation (165 vs $29 \mathrm{~h}$ ), and in the multivariate analysis the need for supplemental oxygen at baseline was the strongest predictor for mortality. Ison summarized that both treatment regimens of peramivir appeared safe and welltolerated, but that the number of patients with virological end points was too small to make reliable conclusions between treatment arms.

\section{Influenza vaccines}

In an overview of vaccines, Albert Osterhaus from the Erasmus University, Rotterdam, Netherlands, commented that the ferret model proved to be the most suitable animal model to both reproduce the pathogenesis of $\mathrm{H} 5 \mathrm{~N} 1$ and $\mathrm{pH} 1 \mathrm{~N} 1$ in humans and to test classical and novel vaccines. Oil-in-water adjuvants have become the current method of choice to increase and broaden vaccine-associated antibody responses and to increase the longevity of the priming effect. These adjuvants have been used in prepandemic $\mathrm{H} 5 \mathrm{~N} 1$ and $\mathrm{pH} 1 \mathrm{~N} 1$ vaccines without causing an unacceptable increase in side effects. In addition, live-attenuated, virus-like particle and vectored vaccines have been successfully tested in animal models against these two viruses. Osterhaus concluded that these advances in the field of pandemic vaccine development will also have their impact on the development of novel generations of seasonal influenza vaccines that should - especially in high risk individuals - elicit higher, broader and longer protective immune responses.

Alain Moren from EpiConcept, Paris, France, presented results from the European multicenter, case-control study (I-MOVE) of $\mathrm{pH} 1 \mathrm{~N} 1 \mathrm{vac}-$ cine effectiveness in seven European countries. Results suggested protection again laboratoryconfirmed pH1N1 influenza-like illness for the monovalent $\mathrm{pH} 1 \mathrm{~N} 1$ vaccine with a multivariate imputation adjusted vaccine effectiveness estimate of $71.9 \%$ overall (95\% CI: 45.6-85.5) [12], 
although in most countries vaccination campaigns began well after the incidence peak. In future, earlier measurements will be conducted in the influenza season at each study site and more study sites will be included.

During the pandemic, pregnant woman were at increased risk of severe infection, but there was an ongoing debate about the safety of the oil-in-water adjuvanted vaccines used in many countries. Fernanda Tavares of the GSK's Global Vaccine Development described a prospective observational, multicenter cohort study including 9000 subjects vaccinated with an AS03adjuvanted $\mathrm{pH} 1 \mathrm{~N} 1$ vaccine. Of these subjects 264 were pregnant when they received the vaccine. No significant differences in the percentage of spontaneous abortions (3.4 vs 3.9-8.6\%), stillbirths ( 0 vs $0.8 \%$ ) or congenital anomalies (1.5 vs $5.5 \%)$ were observed in the vaccinated pregnant women compared with unvaccinated pregnant women (based on historical data from various national statistics or literature).

\section{Acknowledgements}

The authors would like to thank The Macrae Group LLC for organization of the XIII ISRVI and its sponsors: Viroclinics Biosciences (Rotterdam, The Netherlands), Armed Forces Health Surveillance Center (Silver Spring, MD, USA), Department of
Defense Global Emerging Infections System (Silver Spring, MD, USA), Life Technologies (Carlsbad, CA, USA), GlaxoSmithKline (Philadelphia, PA, USA), F Hoffmann-La Roche Ltd. (Basel, Switzerland), Wellcome Trust (London, UK) and The Macrae Group LLC (New York, NY, USA).

\section{Disclosure}

The findings and conclusions in this report are those of the authors and do not necessarily represent the views of the funding agency.

\section{Financial \& competing interests disclosure}

Since 2008, FG Hayden has served as unpaid consultant to multiple companies involved in influenza antiviral and vaccine development. ADME Osterhaus is head of the Department of Virology of Erasmus MC Rotterdam and part-time CSO of Viroclinics-Biosciences BV, a spin-off CRO of Erasmus $M C$ that collaborates with pharmaceutical companies. A Monto reports receiving consultation fees from GlaxoSmithKline, Novartis, Roche and Biocryst, and grant support from SanofPasteur. The authors have no other relevant affiliations or financial involvement with any organization or entity with a financial interest in or financial conflict with the subject matter or materials discussed in the manuscript apart from those disclosed.

No writing assistance was utilized in the production of this manuscript.

\section{Bibliography}

1. Vijaykrishna D, Smith GJD, Pybus OG et al. Long-term evolution and transmission dynamics of swine influenza A virus. Nature 473, 519-522 (2011).

2. Vijaykrishna D, Poon LLM, Zhu HC et al. Reassortment of pandemic H1N1/2009 influenza A virus in swine. Science 328, 1529 (2010).

3. Nelson MI, Lemey P, Tan Y et al. Spatial dynamics of human-origin $\mathrm{H} 1$ influenza $\mathrm{A}$ virus in north american Swine. PLoS Pathog. 7, E1002077 (2011).

4. Fusaro A, Monne I, Salviato A et al. Phylogeography and evolutionary history of reassortant $\mathrm{H} 9 \mathrm{~N} 2$ viruses with potential human health implications. J. Virol. 85(16), 8413-8421 (2011).

5. Cattoli G, Milani A, Temperton $\mathrm{N}$ et al. Antigenic drift in $\mathrm{H} 5 \mathrm{~N} 1$ avian influenza in poultry is driven by mutations in major antigenic sites of the hemagglutinin molecule analogous to human influenza. J. Virol. 85(17), 8718-8724 (2011).
6. Sharshov K, Silko N, Sousloparov I, Zaykovskaya A, Shestopalov A, Drozdov I. Avian influenza (H5N1) outbreak among wild birds, Russia, 2009. Emerg. Infect. Dis. 16, 349-351 (2010).

7. Viboud C, Miller M, Olson D, Osterholm M, Simonsen L. Preliminary estimates of mortality and years of life lost associated with the $2009 \mathrm{~A} / \mathrm{H} 1 \mathrm{~N} 1$ pandemic in the US and comparison with past influenza seasons. PLoS Curr. RRN1153 (2010).

8. Shrestha SS, Swerdlow DL, Borse RH et al. Estimating the burden of 2009 pandemic influenza A (H1N1) in the United States (April 2009-April 2010). Clinical infectious diseases. Clin Infect Dis. 52(Suppl. 1), S75-S82 (2011).

9. de Jong MD, Simmons CP, Thanh TT et al. Fatal outcome of human influenza A (H5N1) is associated with high viral load and hypercytokinemia. Nat. Med. 12, 1203-1207 (2006).

10. Sugaya N, Ohashi Y. Long-acting neuraminidase inhibitor laninamivir octanoate (CS-8958) versus oseltamivir as treatment for children with influenza virus infection. Antimicrob. Agents Chemother. 54, 2575-2582 (2010).

11. Watanabe A, Chang SC, Kim MJ, Chu DWS, Ohashi Y. Long-acting neuraminidase inhibitor laninamivir octanoate versus oseltamivir for treatment of influenza: a double-blind, randomized, noninferiority clinical trial. Clin. Infect. Dis. 51, 1167-1175 (2010).

12. Valenciano M, Kissling E, Cohen JM et al. Estimates of pandemic influenza vaccine effectiveness in Europe, 2009-2010: results of Influenza Monitoring Vaccine Effectiveness in Europe (I-MOVE) multicentre case-control study. PLoS Med. 8, E1000388 (2011).

\section{Website}

101. WHO. Influenza at the human-animal interface www.who.int/influenza/human_animal_ interface/en 